\title{
Rugby Ball Cardiac Calcification
}

\author{
Shreetal Nair ${ }^{1}$ and Sheena $\mathrm{P}^{2}$ \\ ${ }^{1}$ Meitra Hospital \\ ${ }^{2}$ Starcare Hospital
}

April 28, 2020

\begin{abstract}
A 65 year old gentleman, poorly compliant with medications and history of anterior wall myocardial infarction many years back presented with features of acute heart failure. Chest skiagram revealed a peculiar calcification ('Rugby ball') involving the cardiac apex and anterolateral wall. Echocardiography revealed aneurysmal dilatation involving the mid and apical left ventricle with severe left ventricular dysfunction. No mural thrombus was detected.He was stabilized with anti-heart failure medications and advised regular follow up.
\end{abstract}

\section{'Rugby Ball' Cardiac Calcification:}

A 65 year old gentleman presented with features of acute heart failure and on evaluation was found to be in pulmonary edema. He was stabilized with diuretics and other anti-heart failure medications. Historical review revealed acute coronary syndrome almost 15 years back. He had poor compliance with medications. Chest skiagram (Fig 1A; 1B) showed cardiomegaly with a peculiar calcification ('Rugby ball') involving the cardiac apex and anterolateral wall. Echocardiography revealed aneurysmal dilatation involving the mid to apical left ventricle with severe left ventricular systolic dysfunction (Fig 1C; Video - Supplementary file). No mural thrombus was detected. ECG showed features suggestive of previous anterior wall myocardial infarction in the precordial leads. However no persistent ST elevation was noted. He was later discharged after optimization of medications and advised regular follow up. Calcification of ventricular aneurysm is not a common entity. It is recognized that congestive heart failure is the most common presentation of such aneurysms, followed by angina, ventricular arrhythmias, and embolization. Previous reports of calcification of left ventricular aneurysm have been described and management depends on the overall clinical profile ${ }^{1-3}$.

\section{References:}

1. Nakajima O, Sano I, Akioka H. Images in cardiovascular medicine. Marked calcified left ventricular aneurysm. Circulation 1997; 95 (7):1974.

2. Lee BK, Atwood JE. Calcified left ventricular aneurysm. N Engl J Med. 2003; 348:918.

3. Harrison-Gomez C, Harrison-Ragle A, Arceo-Navarro A. Images in cardiovascular medicine. A ring in the heart: calcified left ventricular aneurysm. Circulation 2007;115 (13):e376-7. 

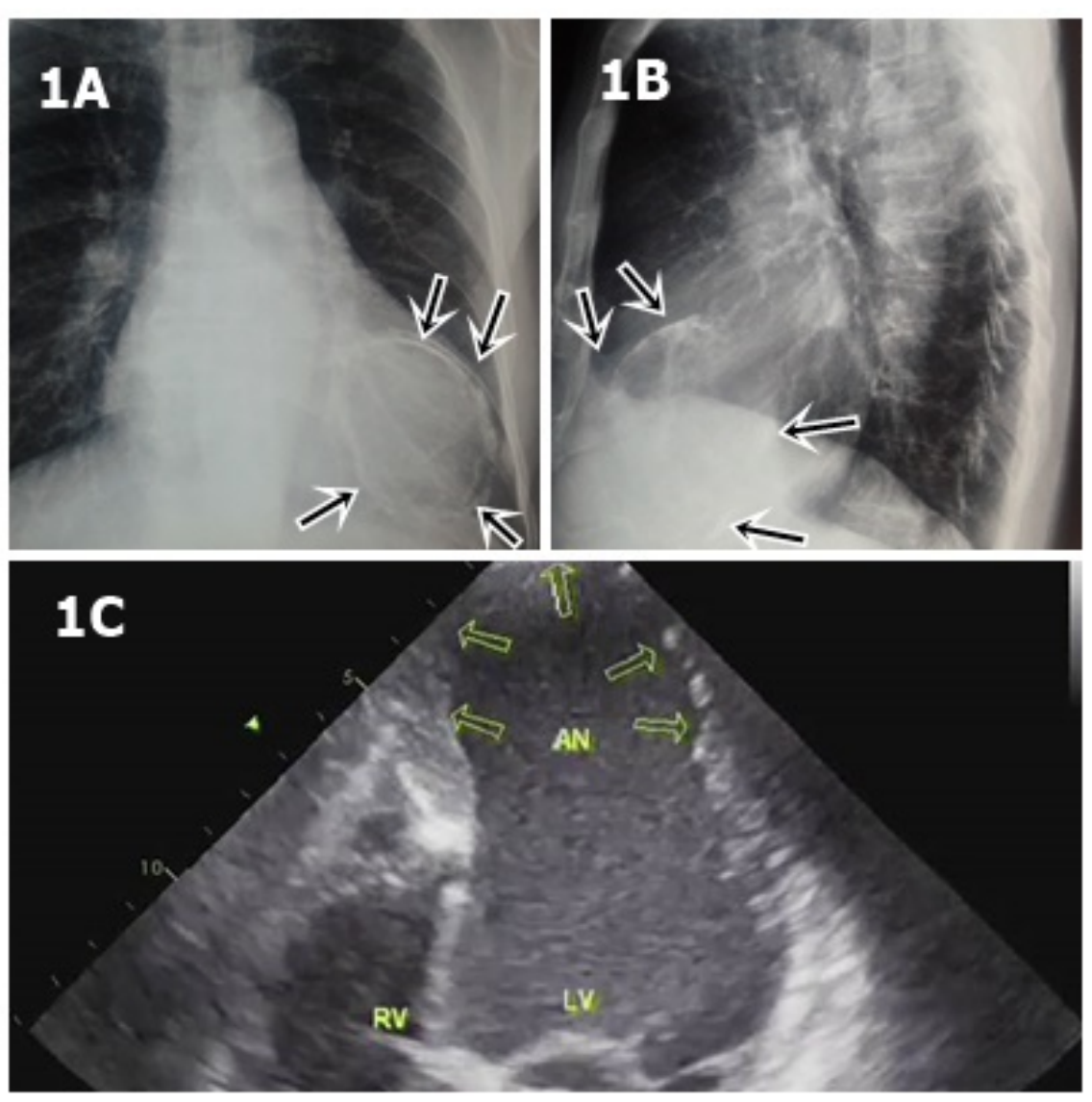